\title{
Noise analysis and optimization of a charge transformer, a noise-matching device for single electron transistors
}

\author{
C. Kurdak ${ }^{\mathrm{a})}$ and K. M. Lewis \\ Department of Physics, University of Michigan, Ann Arbor, Michigan 48109-1120
}

(Received 5 September 2002; accepted 16 December 2002)

\begin{abstract}
Operation and noise analysis of a recently proposed noise-matching device, called a charge transformer, are presented. The charge transformer consists of $N$ identical capacitors and $3 N+1$ switches that enable the capacitors to be connected either in series or in parallel. The device is operated by switching back and forth between these two configurations at speeds faster than the signals that will be measured. We show that an ideal charge transformer can achieve perfect noise matching between any single-electron transistor and a high capacitance device that is under test. We also discuss how a realistic charge transformer made using switches with finite capacitance and resistance should be operated to achieve optimum noise performance. (c) 2003 American Institute of Physics. [DOI: 10.1063/1.1544412]
\end{abstract}

\section{INTRODUCTION}

The single electron transistor (SET) has emerged as a promising device after the discovery of the Coulomb blockade phenomenon arising from discreteness of charge. ${ }^{1-3}$ At present, when used with low-capacitance devices, the charge sensitivity of SET's is superior to all other electrometers, making them essential in applications such as in readout circuitry of charge quantum bits and other metrological and detector applications where low-noise performance is needed. ${ }^{4-6}$ However, when used with high capacitance devices, the noise performance is suppressed due to a capacitance mismatch problem. The problem is more severe for SET's that operate at higher temperatures, since such SET's must have smaller input capacitances. In principle, this problem can be solved using a charge transformer, a recently proposed noise-matching device. A charge transformer consists of multiple capacitors, that are switched back and forth between parallel and series configurations. The charge transformer steps up the input voltage by a factor equal to the number of capacitors used. A prototype charge transformer with four capacitors has recently been demonstrated using the GaAs/AlGaAs heterostructure system. ${ }^{7}$ At present, a charge transformer has not been used with a SET, and improved noise performance has not yet been experimentally demonstrated.

In this article, we discuss fundamental and practical issues that are relevant to the operation of a charge transformer with a low-capacitance electrometer. In the next Sec. II, we introduce the problem of capacitance mismatch for a general electrometer application and then, in Sec. III, discuss the operation of a charge transformer in the context of this mismatch problem. We show that an ideal charge transformer with the right number of capacitors can achieve perfect noise matching between any low-capacitance electrometer and a high-capacitance detector. We analyze a charge transformer with parasitic capacitances, which can degrade the performance of a real charge transformer, especially in applications

\footnotetext{
a)Electronic mail: kurdak@umich.edu
}

where high-voltage step-up ratios are needed. We argue that in situations where parasitic capacitances are significant, a better approach for noise matching is to use multiple charge transformers with fewer capacitors and cascade them to achieve the required voltage step-up ratio. We also discuss the intrinsic noise that is generated by the operation of a charge transformer. The dominant noise source is expected to arise from the unevenness of charge distribution between different capacitors during the operation of the charge transformer. The magnitude of the noise power is found to be inversely proportional to the frequency at which the switches of the charge transformer are operated. We believe a charge transformer or a cascade of charge transformers operating at high frequencies with realistic semiconductor switches can be integrated with SET's and provide significant noise reduction in high-capacitance applications.

\section{CAPACITANCE MATCHING}

Electrometers are typically used to measure charges on high impedance devices or detectors. To illustrate the importance of capacitance matching in an electrometer circuit, we will represent the input of the electrometer and the device under test by capacitors $C_{E}$ and $C_{D}$, respectively, and define the total charge that is generated in the detector, which needs to be measured by the electrometer, as $Q_{\text {Total }}$. For example, in a reverse biased $p-n$ diode detector, $Q_{\text {Total }}$ would be equal to the electron-hole pairs generated by the incident light. If we connect the detector directly to the electrometer, the total charge would split between the electrometer and the detector as follows:

$$
\begin{aligned}
& Q_{D}=\frac{C_{D}}{C_{D}+C_{E}} Q_{\text {Total }}, \\
& Q_{E}=\frac{C_{E}}{C_{D}+C_{E}} Q_{\text {Total }},
\end{aligned}
$$

where $Q_{D}$ and $Q_{E}$ are the charge on the detector and the electrometer, respectively. The total energy that is coupled to the electrometer is 


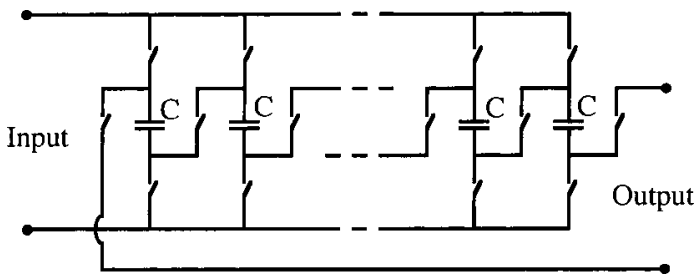

FIG. 1. Circuit diagram of a charge transformer, which consists of $N$ capacitors and $3 N+1$ switches.

$$
U_{E}=\frac{C_{E}}{2\left(C_{D}+C_{E}\right)^{2}} Q_{\text {Total }}^{2}
$$

which has a maximum when $C_{E}=C_{D}$. Thus, if the electrometer used is matched to the detector under test a maximum power of $U_{E, \max }=Q_{\text {Total }}^{2} / 8 C_{D}$ will be coupled to the electrometer. On the other hand, if the input capacitance of the electrometer is much smaller than the detector capacitance $\left(C_{E} \ll C_{D}\right)$, there is a severe mismatch problem and a very small fraction of the energy would be coupled to the electrometer.

Even though the energy sensitivity of SET electrometers can be made close to the quantum limit of $\hbar$, because of this matching problem, it has not yet been advantageous to use SET's for amplifying signals from real macroscopic detectors. In principle, multiple SET's can be used in parallel to solve this problem. Unfortunately, this is not practical, since the operation of multiple real SET's in parallel would require an individual feedback circuit for each SET to compensate the effects of offset charges that are present near the active region of the SET's. ${ }^{8}$ The charge transformer that we will discuss in detail next presents a solution to this mismatch problem.

\section{CHARGE TRANSFORMER AS A CAPACITANCE MATCHING DEVICE}

\section{A. Operation of an ideal charge transformer}

The goal of the charge transformer is to effectively couple the energy from a high-capacitance detector to a lowcapacitance electrometer. A schematic diagram of a recently proposed charge transformer circuit consisting of $N$ identical capacitors of capacitance $C$ and $3 N+1$ switches is shown in Fig. $1 .^{7}$ The switches enable the capacitors to be connected in parallel or in series. In operation, the charge transformer will be placed between the electrometer and device under test and the configuration of the switches will be controlled in cyclic manner as shown in Fig. 2. If $N$ and $C$ are chosen such that in parallel and series configurations the equivalent capacitance of the charge transformer is close to that of the detector under test and the electrometer, respectively, the goal of effective energy transfer from the detector to the electrometer can be achieved.

To quantify how effectively the energy can be transferred from the detector to the electrometer using the charge transformer, let us start with an initial condition where only the detector is charged with a charge of $Q_{\text {Total }}$. Once the cyclic operation starts some of the charge will move from the

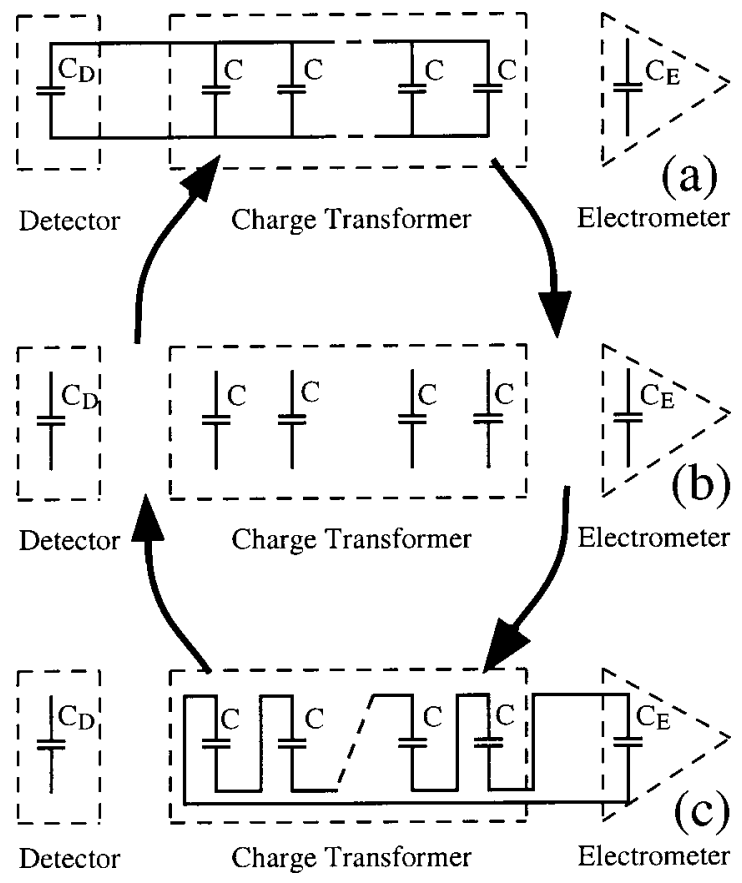

FIG. 2. Different capacitor configurations of a charge transformer during its operation: (a) The configuration where the charge transformer is connected to the detector and all the capacitors are connected in parallel. (b) The configuration where the charge transformer is neither connected to the electrometer nor the detector and all the capacitors are disconnected from each other. (c) The configuration where the charge transformer is connected to the electrometer and all the capacitors are connected in series.

detector to the charge transformer and the electrometer. At a given instant, the state of our system can be described by three parameters; the charges on the detector, charge transformer, and the electrometer. If we assume that no additional charge will be generated in the detector during the cyclic operation of the charge transformer, we can write a charge conservation equation

$$
Q_{\text {Total }}=Q_{D}+N Q+N Q_{E},
$$

where $Q$ is the charge on each capacitor of the charge transformer. Note that the last term is $N Q_{E}$ instead of $Q_{E}$, because the electrometer charges at the expense of $N$ capacitors discharging as shown in Fig. 2(c). After many cyclic operations, the circuit will reach a steady state where the voltage across an individual capacitor of the charge transformer will be equal to the voltage across the detector and be $N$ times smaller than that of the electrometer;

$$
\begin{aligned}
& \frac{Q_{D}}{C_{D}}=\frac{Q}{C}, \\
& N \frac{Q}{C}=\frac{Q_{E}}{C_{E}} .
\end{aligned}
$$

By solving Eqs. (4), (5), and (6), we find the charge on the electrometer at steady state is

$$
Q_{E}=\frac{Q_{\text {Total }}}{\left(\frac{C_{D}}{N C_{E}}+\frac{C}{C_{E}}+N\right)} .
$$


The charge on the electrometer has a maximum value when $N=\sqrt{C_{D} / C_{E}}$. Thus, the total energy that can be transferred to the electrometer using a charge transformer with optimum number of capacitors is

$$
U_{\max }=\frac{Q_{\text {Total }}^{2}}{8 C_{D}}\left(\frac{1}{1+C / 2 \sqrt{C_{D} C_{E}}}\right)^{2} .
$$

The equation suggests that a low-capacitance electrometer used with an optimum charge transformer with $C \leqslant \sqrt{C_{D} C_{E}}$ can access the energy of a high-capacitance detector with an efficiency close to that of an electrometer with matched capacitance.

It is also instructive to do the transient analysis of this problem for a charge transformer with an optimum number of capacitors. Let us represent the state of the circuit after $n$ cycles by $Q_{D, n}, Q_{n}$, and $Q_{E, n}$, which are the charges on the detector, charge transformer, and the electrometer, respectively. Following through the evolution of the charges through a single operation cycle of the charge transformer, we get the following recursion relationship:

$$
\begin{aligned}
\left(\begin{array}{c}
Q_{D, n+1} \\
Q_{n+1} \\
Q_{E, n+1}
\end{array}\right)= & \left(\begin{array}{ccc}
\alpha & \alpha N_{\text {op }} & 0 \\
(1-\alpha)^{2} / N_{\text {op }} & (1-\alpha)^{2} & (1-\alpha) \\
\alpha(1-\alpha) / N_{\text {op }} & \alpha(1-\alpha) & \alpha
\end{array}\right) \\
& \times\left(\begin{array}{c}
Q_{D, n} \\
Q_{n} \\
Q_{E, n}
\end{array}\right)
\end{aligned}
$$

where $\alpha=1 /\left(1+C / \sqrt{C_{D} C_{E}}\right)$ and $N_{\text {op }}=\sqrt{C_{D} / C_{E}}$. If we start with an initial condition of where only the detector is charged with a charge of $Q_{\text {Total }}$, we get

$$
Q_{E, n}=\frac{\alpha\left(1-\alpha^{2 n}\right)}{(1+\alpha) N_{\mathrm{op}}} Q_{\mathrm{Total}} .
$$

In the limit as $n \rightarrow \infty$, the electrometer charge approaches

$$
Q_{E, n \rightarrow \infty}=\frac{Q_{\text {Total }}}{N_{\text {op }}\left(2+C / \sqrt{C_{D} C_{E}}\right)},
$$

with a time constant

$$
\tau=\frac{T_{0}}{2 \ln \left(1+C / \sqrt{C_{D} C_{E}}\right)},
$$

where $T_{0}$ is the period of the charge transformer operation cycle. Note that the asymptotic result for the electrometer charge given in Eq. (11) is consistent with the steady-state result given in Eq. (7). The energy transferred to the electrometer and the charge transfer time are plotted as a function of $C$ in Fig. 3. The charge transfer time is defined as the maximum of $\tau$ and $T_{0}$. This analysis indicates that, in choosing $C$, there is a compromise between a fast electrometer response and a high-energy transfer to the electrometer. Both a fast electrometer response and an efficient energy transfer can be achieved using a charge transformer with $C$ $\approx \sqrt{C_{D} C_{E}}$.

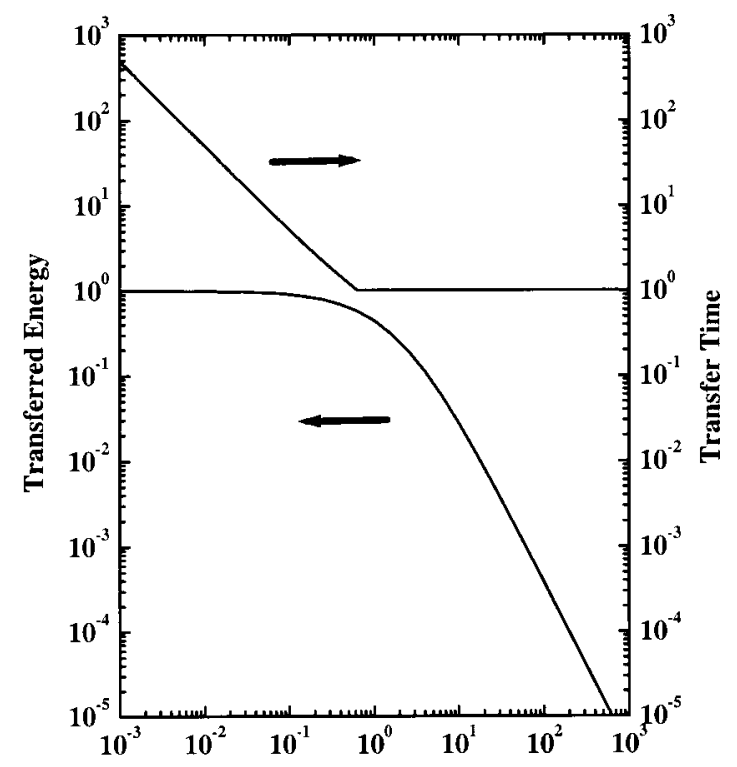

Charge Transformer Capacitance

FIG. 3. Energy transferred to the electrometer by the charge transformer and the charge transfer time vs charge transformer capacitance. The transferred energy, the transfer time, and the charge transformer capacitance are normalized with respect to $Q_{\text {Total }}^{2} / 8 C_{D}, T_{0}$, and $\sqrt{C_{D} C_{E}}$, respectively.

\section{B. Charge transformer with real switches}

So far, we have assumed that the charge transformer is made using ideal switches. However, in a real implementation, the switches will have finite parasitic capacitances, which can significantly degrade the operation of a charge transformer. In the operation cycle of the charge transformer, the effect of the switches is most pronounced when the capacitors are connected in series with the electrometer as shown in Fig. 2(c). In this series configuration, the equivalent circuits of an ideal charge transformer and one with parasitic capacitances are shown in Fig. 4. In the ideal circuit, the equivalent capacitance seen by the electrometer is $C / N$, and the output voltage of the charge transformer is $N$ times that of the input voltage. This step-up ratio is degraded by parasitic capacitance. The problem is most severe for charge transformers with a large number of capacitors. In the limit when $N$ is infinite, the equivalent capacitance seen by the electrometer is

$$
C_{\mathrm{eq}}=\frac{\sqrt{4 C_{p} C+C_{p}^{2}}-C_{p}^{2}}{2} \approx \sqrt{C_{p} C},
$$

where $C_{p}$ is the parasitic capacitance of the switches. The maximum step-up ratio allowed with parasitic capacitances is $\sqrt{C / C_{p}}$.

To illustrate the severity of this problem, let us consider an example where we need to noise match a detector with a $C_{D}=1 \mathrm{nF}$ to an electrometer with $C_{E}=1 \mathrm{fF}$. Ideally, the noise matching can be accomplished with a charge transformer with 1000 capacitors each with a capacitance of $1 \mathrm{pF}$ as shown in Fig 5(a). In this example, if the switches have parasitic capacitance of approximately $1 \mathrm{aF}$ or larger, the step-up ratio and thus the noise matching of the charge transformer would be significantly degraded. Semiconductor 


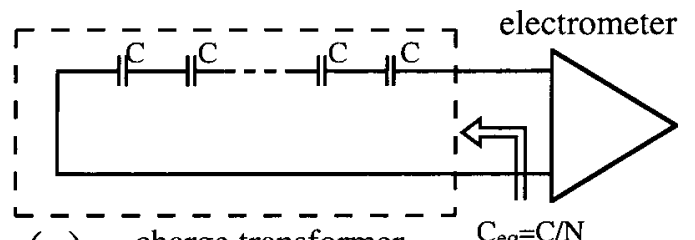

(a)

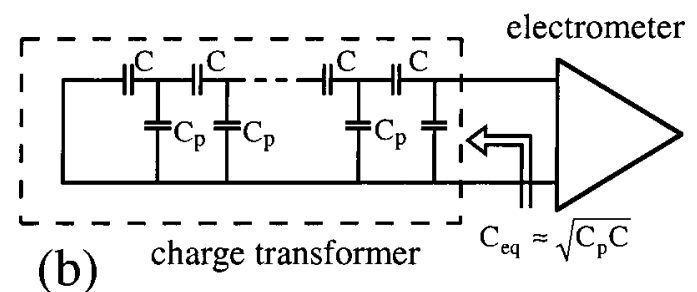

FIG. 4. The equivalent circuits of a charge transformer when connected to an electrometer without and with parasitic capacitances. For large $N$, the equivalent capacitance seen by the electrometer in these two circuits are $C / N$ and $\sqrt{C_{p} C}$, respectively.

switches, even the small ones made by state-of-the-art electron-beam lithography techniques, have parasitic capacitances typically much larger than $1 \mathrm{aF}$. This places a serious constraint on the maximum step-up ratio that one can achieve using a single charge transformer. Alternatively, we can accomplish the same task of noise matching using multiple charge transformers that are operated in a cascade configuration. This approach is less vulnerable to parasitic effects as there are fewer switches and capacitors needed to achieve the same voltage step-up ratio. For instance, to get a step-up ratio of 1000 , we can use three charge transformers each with ten capacitors as shown in Fig. 5(b). Capacitance matching can be achieved using capacitance values of 100 $\mathrm{pF}, 1 \mathrm{pF}$, and $10 \mathrm{fF}$ with parasitic switch capacitances that are smaller than $1 \mathrm{pF}, 10 \mathrm{fF}$, and $100 \mathrm{aF}$ for the first, second, and third charge transformers, respectively. Note that the restrictions on parasitic capacitances are relaxed by a factor of 100 using this approach. Furthermore, cascade operation has an

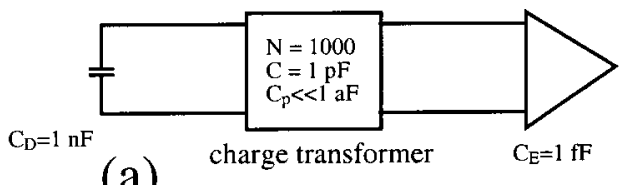

(a)

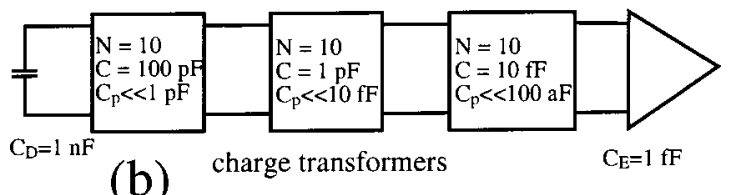

FIG. 5. (a) Block diagram of a noise matching application where a single charge transformer is placed between a $1 \mathrm{nF}$ detector and a $1 \mathrm{fF}$ electrometer. This application requires a voltage step-up ratio of 1000 . If a charge transformer with $N=1000$ and $C=1 \mathrm{pF}$ were to be used, the parasitic capacitances of the switches must be less that $1 \mathrm{aF}$ to achieve this goal. (b) Block diagram of an alternative approach for the same noise matching application where three charge transformers are used. Each charge transformer provides a step-up ratio of 10 leading to a step-up ratio of 1000 for the cascade operation. Since fewer capacitors are needed, the constraint for the maximum allowable switch capacitance is significantly relaxed.

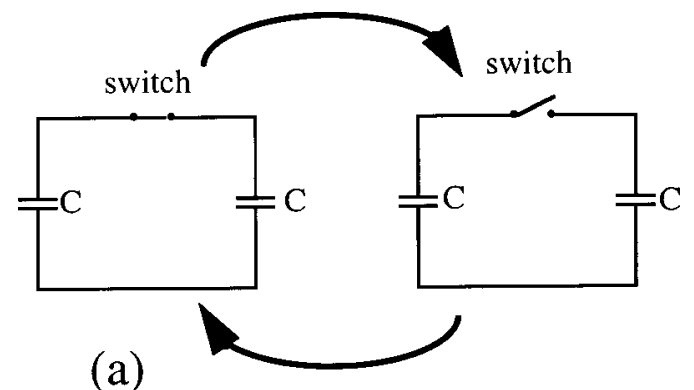

(b)

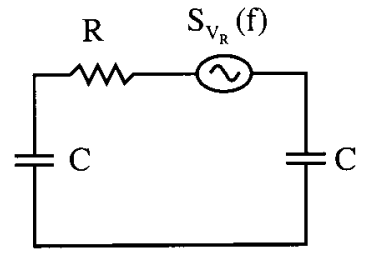

FIG. 6. (a) Diagram showing the cyclic operation of a simple circuit consisting of two identical capacitors and a single switch. (b) The noise equivalent diagram of such a circuit where the switch is replaced by a resistor.

additional advantage in that it reduces the number of components needed to achieve capacitance matching. In this example, one can use 30 capacitors instead of 1000 .

\section{Charge transformer noise}

The operation of a charge transformer is very different from that of other noise matching devices, such as superconducting flux transformers used with superconducting quantum interference devices or conventional transformers that operate on the basis of magnetic induction used with semiconductor amplifiers. The noise properties of a charge transformer have not been studied experimentally or theoretically before. We will discuss noise mechanisms that are unique to the operation of the charge transformer.

The primary noise arises from the fact that charge transformer splits a finite amount of charge between different capacitors, which cannot be done evenly when few electrons are involved. To illustrate the physical origin of this noise, let us start with a simple circuit consisting of two identical capacitors and a single switch. Let us assume the total charge on these two capacitors is $Q_{T}$ and the switch is opened and closed with a period of $T_{0}$ causing the capacitors to go from one configuration to another in a cyclic manner as shown in Fig. 6(a). Note that at a given instant when the switch is open, the charge on one of the capacitors is not necessarily equal to $Q_{T} / 2$. There are two sources of noise in partitioning the charge between the two capacitors. The first source of noise is due to the discrete nature of charge and it is easy to understand; if the total number of electrons on the two capacitors is odd, the charge cannot be split evenly, since each capacitor must have an integer number of electrons. This leads to a root-mean-square charge noise of $e / 2$ on each capacitor for an odd number of total electrons.

The second source of noise is due to thermal fluctuations. To understand why the thermal fluctuations play a role in this problem, we must look at what is happening to the charges in the switching process. Of course, it is unphysical 
to assume that the switch can go from the zero resistance state (closed) to the infinite resistance state (open) instantaneously. Instead, to simulate the process of opening of a switch, let us replace the switch with a resistor as shown in Fig. 6(b) and allow the resistance to increase as a function of time gradually. At a given instant, the circuit can be represented by a resistor $R$ and two capacitors all connected in series. At this instant, the noise power spectrum of the resistor is given by the Nyquist formula, $S_{V_{R}}=4 k T R$, where $k$ is the Boltzmann constant and $T$ is the temperature. The formula is, of course, only meaningful if $R$ is varying slowly. Using the noise equivalent circuit shown in Fig. 6(b), we calculate the noise power spectral function for the voltage across the capacitor

$$
S_{V_{C}}(f)=\frac{1}{2}\left(\frac{1}{1+(\pi f R C)^{2}}\right) S_{V_{R}} .
$$

The mean square voltage noise can be calculated by integrating the noise spectral function

$$
\overline{V_{C}^{2}}=\int_{0}^{\infty} S_{V_{C}}(f) d f=\frac{k T}{C} .
$$

The magnitude of the voltage fluctuations we get from this calculation is consistent with what one expects from the equipartition theorem.

Note that, at some point in time, as we are opening the switch, the approximation that $R$ is varying very slowly must break down. This must happen when the $R C$ time constant becomes comparable to the operating period $T_{0}$. So for $R$ $>T_{0} / C$, we can call the switch open. When the switch is opened, the capacitor charge will be nonzero and this charge must be represented by a random variable with a variance given by equipartition theorem.

The charge on an electrometer connected to a charge transformer will exhibit noise similar to the capacitor in the single switch circuit discussed herein. Noise arising from both the discrete nature of charges as well as thermal fluctuations must be included. We can express charge fluctuations on the electrometer in the time domain by

$$
Q_{E}(t)=Q_{E-S S}+\sum_{m} Q_{E m} u\left(t-m T_{0}\right),
$$

where $Q_{E-S S}$ is the steady-state charge on the electrometer, $Q_{E m}$ is a random variable representing charge noise we get on the $m$ th operation cycle of the charge transformer, and $u(t)$ is a pulse function with a value equal to 1 for $0<t$ $<T_{0}$ and 0 elsewhere. In the worst case scenario, when $Q_{E-S S}$ is a half integer, the variance of the random charge is

$$
\overline{Q_{E l} Q_{E m}}=\left(\frac{1}{4} e^{2}+C_{E} k T\right) \delta_{l, m},
$$

where both sources of noise are included and the Kronecker delta, $\delta_{l, m}$, is used since the charge noise between different operation cycles of the charge transformer is not expected to be correlated. The corresponding autocorrelation function for the electrometer charge noise can be represented by the following piece-wise function

$$
\begin{aligned}
& \overline{Q_{E}(t) Q_{E}\left(t+\tau_{0}\right)}-Q_{E-S S}^{2} \\
& \quad= \begin{cases}\left(\frac{1}{4} e^{2}+C_{E} k T\right)\left(1-\left|\tau_{0}\right| / T_{0}\right) & \text { for }\left|\tau_{0}\right|<T_{0} \\
0 & \text { for }\left|\tau_{0}\right|>T_{0} .\end{cases}
\end{aligned}
$$

The noise power spectral density for the electrometer charge can be calculated from the autocorrelation function using the following integral

$$
\begin{aligned}
S_{Q_{E}}(f) & =\int_{0}^{\infty}\left(\overline{Q_{E}(t) Q_{E}\left(t+\tau_{0}\right)}-Q_{E-S S}^{2}\right) \cos \left(2 \pi f \tau_{0}\right) d \tau_{0} \\
& =\left(\frac{1}{4} e^{2}+C_{E} k T\right) \frac{1-\cos \left(2 \pi f T_{0}\right)}{(2 \pi f)^{2} T_{0}}
\end{aligned}
$$

At low frequencies, $f \ll 1 / T_{0}$, the charge noise as seen by the electrometer, due to the charge transformer is white with an noise amplitude of $S_{Q_{E}} \approx\left(e^{2}+4 C_{E} k T\right) T_{0} / 8$. For a SET electrometer, the thermal noise term must be small in comparison to the first term, since SET's are operated at low temperatures where the charging energy is smaller than thermal energy. ${ }^{9}$ Thus, for a SET electrometer integrated with a charge transformer, the dominant charge noise source will arise from the discreteness of charge, $S_{Q_{E}} \approx e^{2} T_{0} / 8$. Note that this noise is not related to shot noise observed in various electronic devices which also results from the discreteness of charge.

The charge transformer noise can be minimized by operating the charge transformer at high frequencies. Semiconductor switches can be operated at microwave frequencies. A realistic charge transformer with a period of operation $T_{0}$ $=1 \mathrm{~ns}$ would have a charge noise $S_{Q_{E}} \approx 1.2 \times 10^{-10} e^{2} / \mathrm{Hz}$, which can be comparable to the intrinsic noise of a SET.

\section{CONCLUSIONS}

We discussed the operation and noise analysis of a charge transformer in noise matching applications for lowcapacitance electrometers. We show, when the switches are ideal, a charge transformer with the right number of capacitors can achieve perfect noise matching between any lowcapacitance electrometer and a high-capacitance detector. If the switches are not ideal, i.e., if the switches have finite capacitance and resistance, the noise performance can be significantly degraded. We anticipate a particularly serious problem in real applications where high-voltage step-up ratios are needed. We show that in such cases, it is much better to use multiple charge transformers with fewer capacitors and cascade them to achieve the required voltage step-up ratio. We also show that the charge transformer would introduce an intrinsic noise due to charge splitting. This noise, which is unique to a charge transformer, can be suppressed by operating the charge transformer at high frequencies. SET 
electrometers integrated with a charge transformer or a cascade of charge transformers may find useful applications in various fields where low-noise performance is needed. The issues discussed in this article can guide the design of charge transformers for specific applications.

\section{ACKNOWLEDGMENTS}

The authors thank Neil M. Zimmerman for careful reading of the manuscript and useful comments. This work is supported by the Alfred P. Sloan Foundation, Research Corporation, and the NSF through Grant No. DMR-0092726.
${ }^{1}$ D. V. Averin and K. K. Likharev, J. Low Temp. Phys. 62, 345 (1986).

${ }^{2}$ T. A. Fulton and G. J. Dolan, Phys. Rev. Lett. 59, 109 (1987)

${ }^{3}$ Single Charge Tunneling, edited by H. Grabet and M. H. Deverot (Plenum, New York, 1992), p. 1.

${ }^{4}$ A. Aassime, G. Johansson, G. Wendin, R. J. Schoelkopf, and P. Delsing, Phys. Rev. Lett. 86, 3376 (2001).

${ }^{5}$ S. Komiyama, O. Astafiev, V. Atanov, T. Kutsuwa, and H. Hirai, Nature (London) 403, 405 (2000).

${ }^{6}$ J. M. Martinis, M. Nahum, and H. D. Jensen, Phys. Rev. Lett. 72, 904 (1994).

${ }^{7}$ K. M. Lewis, Ç. Kurdak, S. Krishna, and P. Bhattacharya, Appl. Phys. Lett. 80, 142 (2002).

${ }^{8}$ G. Zimmerli, T. M. Eiles, R. L. Kautz, and J. M. Martinis, Appl. Phys. Lett. 61, 237 (1992).

${ }^{9}$ N. M. Zimmerman and M. W. Keller, J. Appl. Phys. 87, 8570 (2000). 